\title{
Corrigendum: Strong ferromagnetism at the surface of an antiferromagnet caused by buried magnetic moments
}

A. Chikina, M. Höppner, S. Seiro, K. Kummer, S. Danzenbächer, S. Patil, A. Generalov, M. Güttler, Yu. Kucherenko, E.V. Chulkov, Yu. M. Koroteev, K. Koepernik, C. Geibel, M. Shi, M. Radovic, C. Laubschat \& D.V. Vyalikh

Nature Communications 5:3171 doi: 10.1038/ncomms4171 (2014); Published 21 Jan 2014; Updated 26 Feb 2014

The original version of this Article contained a typographical error in the spelling of the author K. Koepernik, which was incorrectly given as K. Köpernik. This has now been corrected in both the PDF and HTML versions of the Article. 